\title{
基于海水介质的微型面接触摩擦副应力及 摩擦学研究"
}

\author{
周 杰 $^{1}$ 吴进军 ${ }^{1}$ 于革刚 ${ }^{1}$ 杨友胜 $^{2}$ 段海涛 $^{3}$ 易 蒙 $^{3}$ \\ (1. 机械科学研究总院产业发展部 北京 100044; \\ 2. 中国海洋大学工程学院 青岛 266100; \\ 3. 武汉材料保护研究所摩擦学实验室 武汉 430030)
}

\begin{abstract}
摘要: 为了研究微型面接触摩擦副在海水环境中的动力学和摩擦学性能, 根据柱塞运动过程无刚性冲击和柔性冲击的准则, 选取了 $\mathrm{B}$ 样条曲线、五次多项式曲线和正弦曲线作为研究对象, 计算了微型面接触摩擦副接触应力以及摩擦应力; 以动力学 计算的结果对摩擦试验进行加载, 笁选摩擦副材料。结果表明三种曲线组成的摩擦副接触应力最大值约为 $160 \mathrm{MPa}$, 摩擦应 力最大值均约为 $16 \mathrm{MPa}, \mathrm{B}$ 样条曲线和正弦曲线综合力学性能优于五次多项式曲线。通过销盘摩擦试验考察了在初始接触 应力约为 $160 \mathrm{MPa}$, 滑动速度为 $0.5 \mathrm{~m} / \mathrm{s}$ 的工况下, 纯 PEEK、含有 $30 \%$ 玻璃纤维的 PEEK 和分别含有 $10 \%$ 石墨/碳纤维/PTFE 的 PEEK 与 $17-4 \mathrm{PH}$ 组成摩擦副的摩擦学性能。试验结果表明分别含有 10\%石墨/碳纤维/PTFE 的 PEEK 与 17-4PH 摩擦副的 摩擦因数和磨损量均最小, 磨损机理为轻微的粘着磨损, 可作为微型面接触摩擦副滚动体材料。
\end{abstract}

关键词: 微型面接触摩擦副; 接触应力; 摩擦应力; 聚醚醚酮; 海水径向柱塞原

中图分类号: TG122

\section{Study on Stress and Tribology of Micro Contact Surface in Seawater}

\section{ZHOU Jie $^{1}$ WU Jinjun $^{1} \quad$ YU Gegang $^{1} \quad$ YANG Yousheng $^{2} \quad$ DUAN Haitao $^{3} \quad$ YI Meng $^{3}$}

(1. Industrial Development Department, China Academy of Machinery Science \& Technology, Beijing 100044;

2. College of Engineering, Ocean University of China, Qingdao 266100;

3. Tribology Laboratiry, Wuhan Research Institute of Materials Protection, Wuhan 430030)

\begin{abstract}
In order to study the dynamic and tribological performance of micro contact surface in seawater, by the goals of no stiff impact and flexible impact, study focus on the contact pressure and tribological stress of B-spline curve, quantic polynomial curve and sine curve, and the friction experiments were carried out, with the results to select the material of friction pair. The results shows that the maximum contact stress is about $160 \mathrm{MPa}$, the maximum tribological stress is about $16 \mathrm{MPa}$. Moreover, the mechanical performance of B-spline curve and sine curve is better than quantic polynomial curve. The tribological behavior, of three kinds PEEK composites (pure PEEK, 30\% glass fiber PEEK and 10\% graphite/10\% carbon fiber/10\%PTFE PEEK) coupled with $17-4 \mathrm{PH}$, were compared under seawater by pin-on-disc friction test, the operating condition is about $160 \mathrm{MPa}$ and $0.5 \mathrm{~m} / \mathrm{s}$, results indicates that the wear and friction coefficient of $10 \%$ graphite $10 \%$ carbon fiber/10\%PTFE PEEK and $17-4 \mathrm{PH}$ friction pair is minimum, the main mechanism is light abrasive wear, so $10 \%$ graphite $/ 10 \%$ carbon fiber $10 \%$ PTFE PEEK composite is suitable for micro contact surface.
\end{abstract}

Key words: micro contact surface; contact stress; tribology stress; polyether-ether-ketone; seawater radial piston pump

\section{0 前言}

目前反渗透海水淡化主要方式是通过电力或者 内燃机等动力形式, 驱动轴向海水柱塞葲加压海水,

* 国家自然科学基金资助项目(51475197)。20170318 收到初稿, 20170918 收到修改稿
透过半透膜产生淡水。但是在一些偏远海岛, 缺乏 电力等常规动力, 无法提供轴向柱塞洜所需要的高 转速。而这些地区潮流能、潮汐能、波浪能等海洋 能资源丰富, 通过简单的转化即可获得动力。但是 这种动力产生的转速低, 小于 $100 \mathrm{r} / \mathrm{min}^{[1]}$, 因此急 需研制能在低转速下进行反渗透海水淡化的海水 泵。为了解决以上问题, 机械科学研究总院和中国 
海洋大学所组成的国家自然基金项目组提出了低速 海水径向柱塞洜的构想(结构如图 1 所示)。这种海 水葲采用径向式(指柱塞排列方式)代替轴向式或卧 式, 通过增加单转柱塞往复运动频率, 加快柱塞运 动速度, 补偿因压差产生的泄漏, 提高㬌在低转速 下的容积效率。

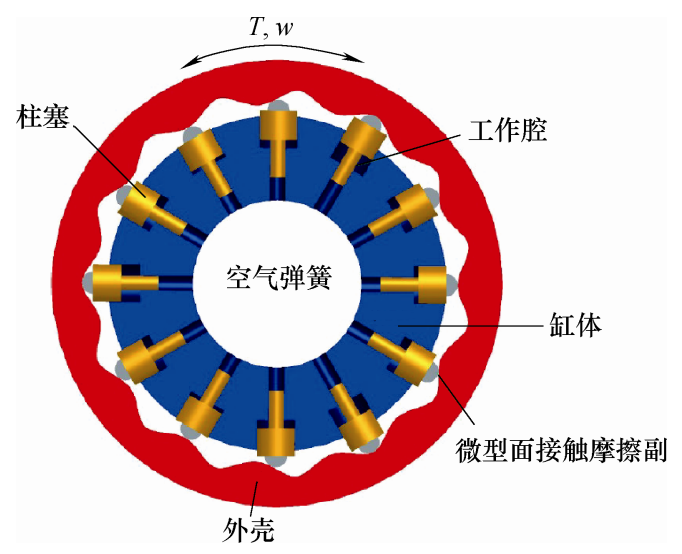

图 1 低速海水径向柱塞百工作原理图

高殿荣等 ${ }^{[2]}$ 对幅角修正等加速运动规律和匀变 加速修正等加速运动规律的加速度公式进行了修 正, 设计了 9 种不同类型运动规律的曲线, 并分析 了柱塞的加速度变化规律。黄瑶 ${ }^{[3]}$ 应用 Hertz 接触理 论和 ANSYS 静力学计算了微型面接触摩擦副的接 触应力。胡波 ${ }^{[4]}$ 对径向海水柱塞泵整机结构进行 了设计和计算, 并分析了柱塞的运动和阀的流量 特性。翟汇 ${ }^{[5]}$ 计算了轴向柱塞泵柱塞副、滑靴副 和配流副的 PV 值, 并考察了海水环境中聚醚醚酮 (Polyetheretherketone, PEEK)复合物与金属摩擦副 的摩擦学性能。杨曙东 ${ }^{[6]}$ 的研究集中在轴向柱塞泵 关键摩擦副的结构设计, 他还考察了陶瓷与金属、 碳纤维增强高分子复合材料与金属摩擦副在海水工 况下的摩擦学性能。YIN 等 ${ }^{[7]}$ 研究了轴向柱塞泵空 化的影响, 以及聚酰亚胺(Polyimide, PI)、聚醚醚 酮复合物与金属配对摩擦副在海水环境中的摩擦磨 损特性。ZHANG 等 ${ }^{[8]}$ 对轴向海水柱塞泵的退化进行 了预测, 通过盘对盘摩擦试验研究了 PI 和 PEEK 复 合材料与不同金属配对时的摩擦学特点。刘恒龙 等 ${ }^{[9]}$ 设计了三腔独立支承的滑靴副结构, 增加了滑 靴副抗倾覆能力, 有效降低了滑靴副偏磨现象。

综上可以看出, 高分子材料在水压元件的应用 已较为广泛, 尤其是 PEEK 材料, 其优异的摩擦学 性能已被认同, 因此本文选择 PEEK 复合材料作为 微型面接触摩擦副材料。

以往对于摩擦副的研究主要针对于海水环境中 滑动摩擦副; 对于海水径向柱塞泵 (马达), 研究内 容集中于结构设计、流场分析、动力学分析和配流
副等方面。微型面接触摩擦属于高副接触, 接触应 力和摩擦应力极高, 以往的研究结论不能完全适用。 在海水环境中, 无法使用固体润滑或者油脂润滑, 因而极其容易产生严重的磨损，可能直接导致水泵 失效, 这也是至今没有做出样机的主要原因。因此 有必要对微型面接触摩擦副进行深入的研究, 揭示 水基条件下高副的应力和摩擦学特点, 通过试验考 察其摩擦学特性, 笁选海水环境中微型面接触摩擦 副材料。

本文将从瞬态动力学入手分析不同形状的外壳 内曲线和滚动体之间的接触应力与摩擦应力, 在此 基础上设计摩擦试验，考察几种 PEEK 复合物与金 属摩擦副摩擦学性能, 笁选出适合的摩擦副材料。

\section{1 微型面接触摩擦副瞬态动力学分析}

为了避免运动过程中柱塞的柔性冲击和刚性 冲, 加速和速度曲线平滑过渡, 没有奇点, 选取 $\mathrm{B}$ 样条曲线、正弦曲线、五次多项式曲线作为微型面 接触摩擦副的内曲线(见图 2$)^{[4]}$ 。

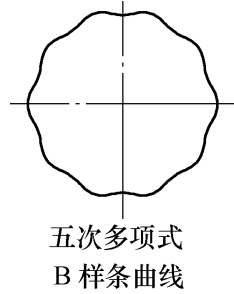

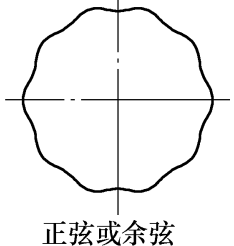

正弦曲线

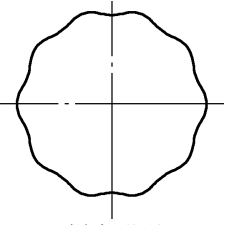

$\mathrm{B}$ 样条曲线 五次多项式曲线
图 2 内曲线形状

内曲线与滚动体组成的微型面接触摩擦的接触 应力和摩擦应力副通过 ANSYS Workbench 平台中瞬 态动力模块 Transient Structural 求解。基本求解原理为

$$
\boldsymbol{M} \cdot \ddot{\boldsymbol{u}}(t)+\boldsymbol{C} \cdot \dot{\boldsymbol{u}}(t)+\boldsymbol{D} \cdot \boldsymbol{u}(t)=\boldsymbol{F}(t)
$$

式中, $\boldsymbol{M}$ 为系统结构质量矩阵; $\boldsymbol{C}$ 为系统结构阻尼 矩阵; $\boldsymbol{D}$ 为系统刚度矩阵; $\ddot{\boldsymbol{u}}(t)$ 为系统加速度矩阵; $\dot{\boldsymbol{u}}(t)$ 为系统速度矩阵; $\boldsymbol{u}(t)$ 为系统位移矩阵; $\boldsymbol{F}(t)$ 为 系统所受外力。

式(1)是瞬态分析的一般形式, 也是 Transient Structural 求解的基本方程, 用于确定承受任意的随 时间变化载荷结构的动力学响应, 可求解确定结构 在稳态载荷、瞬态载荷和简谐载荷的随意组合作用 下随时间变化的位移、应变、应力等。

为了减少计算量, 对模型进行简化, 模型保留 一个柱塞副, 外圈和缸体也简化到只保留与柱塞相 接触的部分; 滚动体为 $\Phi 18 \mathrm{~mm} \times 29 \mathrm{~mm}$ 圆柱, 由于 模型沿滚动体轴向对称, 所以模型采用对称分析, 如图 3 所示。柱塞副与缸体的摩擦副和滚动体与柱 
塞的摩擦副都属于低副, 工况与轴向柱塞泵相近, 根据已有研究摩擦因数取 $0.1^{[8-9]}$ 。微型面接触摩擦 副接触面接触应力极高, 接触面之间很难形成水膜 或者形成的水膜厚度只有几十纳米, 摩擦因数取 $0.1^{[10-11]}$ 。运动设置外壳固定, 䍂体转动速度为 6.5 $\mathrm{rad} / \mathrm{s}$, 加载压力 $1500 \mathrm{~N}$, 缸体设为 Rigid, 滚动体 材料按照 PEEK 典型参数设置, 其余材料按照 17-4PH 材料参数设置。计算时长设为 $0.05 \mathrm{~s}$, 即计 算排水过程中滚动体与外圈的接触应力和摩擦应 力, 时间步长最大为 $0.001 \mathrm{~s}$, 最小为 $0.0005 \mathrm{~s}$ 。

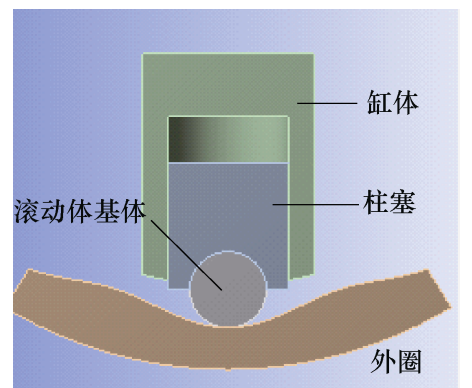

图 3 低速海水径向柱塞泵微型面接触摩擦副模型

接触面使用 Refinement 细化外圈和滚动体网 格, 滚动体的最小网格尺寸约为 $0.4 \mathrm{~mm}$ 。样条曲线 模型网格数为 90145 , 节点数 52766 ; 多项式曲线 模型网格数为 79739 , 节点数 45815 ; 正弦曲线模 型网格数为 88461 , 节点数 52168 。

图 4 和表 1 为三种内曲线和滚动体接触时的摩

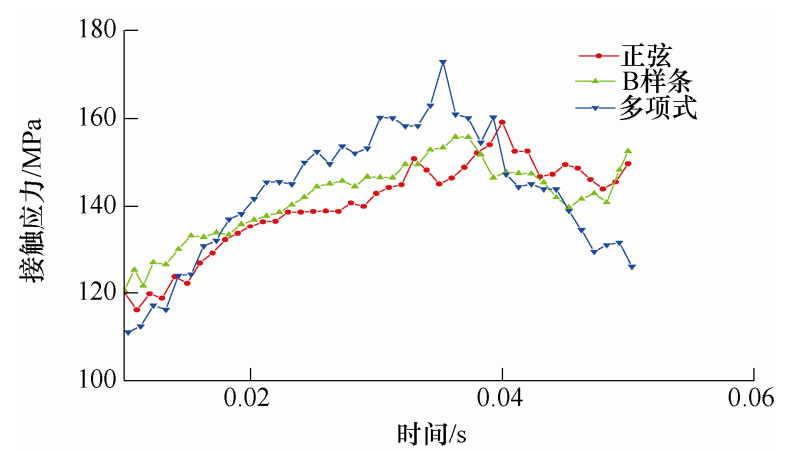

(a) 接触应力

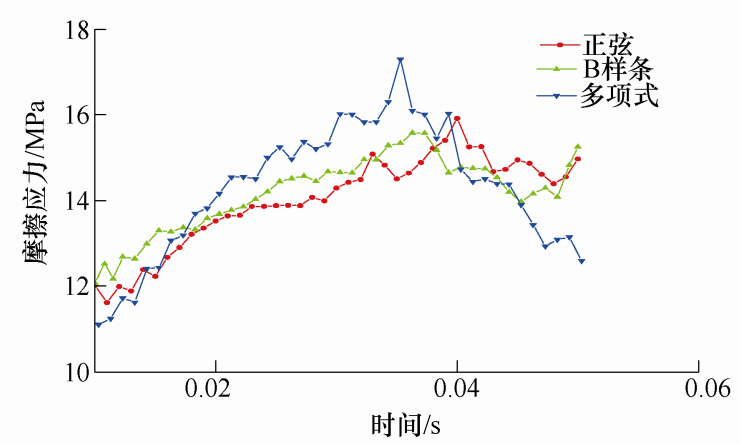

(b) 摩擦应力

图 4 应力随时间变化规律
擦应力和接触应力计算结果, $\quad 0 \sim 0.01 \mathrm{~s}$ 时滚动体 在压力作用下开始变形, 应力波动较大, 与实际 工况差别较大, 因此在分析时将 $0 \sim 0.01 \mathrm{~s}$ 的数据 剔除。

表 1 接触应力和摩擦应力

\begin{tabular}{|c|c|c|c|c|c|c|c|c|}
\hline & \multicolumn{4}{|c|}{ 摩擦应力/MPa } & \multicolumn{4}{|c|}{ 接触应力 $/ \mathrm{MPa}$} \\
\hline & 平均值 & 标准差 & 最小值 & 最大值 & 平均值 & 标准差 & 最小值 & 最大值 \\
\hline 正弦 & 14.01 & 1.08 & 11.61 & 15.91 & 140.14 & 10.84 & 116.13 & 159.13 \\
\hline 样条 & 14.16 & 0.91 & 12.06 & 15.58 & 141.60 & 9.07 & 120.63 & 155.77 \\
\hline 多项式 & 14.28 & 1.51 & 11.10 & 17.28 & 142.84 & 15.11 & 111.04 & 172.84 \\
\hline
\end{tabular}

从图 4 和表 1 可以看出接触应力和摩擦应力大 小随着内曲线的曲率半径的减小而增大。三种曲线 中模型平均接触应力和摩擦应力的次序为 $\mathrm{B}$ 样条 $>$ 正弦>多项式; 标准差的次序与应力次序相同, 即多项式曲线应力值波动最大。从图 4 可以看出多 项式的曲线的应力值在 $0.03 \sim 0.05 \mathrm{~s}$ 之间起伏较 大, 这种波动会加速微型面接触摩擦副的疲劳磨 损。正弦曲线与样条曲线的各项指标相近, 摩擦应 力和接触应力的最大值、标准差等指标明显优于多 项式曲线。

从表 1 中可知, 正弦曲线和样条曲线模型的微 型面接触摩擦副最高接触应力接近 $160 \mathrm{MPa}$ ，摩擦 应力最大值接近 $16 \mathrm{MPa}$ 。而已有的水基润滑下聚合 物与金属摩擦副研究, 大多属于低副摩擦, 接触应 力小于 $10 \mathrm{MPa}$, 显然微型面接触摩擦副结构对摩擦 副材料的要求更加苛刻。

因此, 必须进行材料摩擦学性能测试, 选择摩 擦副材料, 进而实现设计功能。

\section{2 摩擦试验}

\section{1 试验材料}

PEEK 具有密度小、易成形、耐腐蚀等优点, 尤其在海水环境中, 由于海水的冷却和冲刷作用, 带走大部分热量和磨屑, 在一定程度上抑制了材料 的磨损 ${ }^{[12]}$, PEEK 吸水性较好, 也容易形成边界润 滑层 ${ }^{[13]}$, 所以在海水泵摩擦副中已广泛应用。

参考已有的研究 ${ }^{[14]}$, 试验选择了以下三种聚合 物材料作为试验材料: 纯 PEEK 树脂热压板材 (以下 简称 PEEK 1), 含 $30 \%$ 玻璃纤维的 PEEK 热压板材 (以 下简称 PEEK2), 分别含短碳纤维、PTFE、石墨各 $10 \%$ 的 PEEK 热压板材(以下简称 PEEK3), 以上材 料均从常州君华特种工程塑料制品有限公司购得。 具体参数指标如表 2 (洛氏硬度值为样品实测数据, 其余参数为厂家提供)。 
表 2 三种材料的参数

\begin{tabular}{lccc}
\hline & PEEK1 & PEEK2 & PEEK3 \\
\hline 密度 $/\left(\mathrm{g} / \mathrm{cm}^{3}\right)$ & 1.3 & 1.51 & 1.44 \\
吸水率 $\left(25^{\circ} \mathrm{C}, 24 \mathrm{Hrs}\right)(\%)$ & 0.5 & 0.11 & 0.06 \\
熔点 $/{ }^{\circ} \mathrm{C}$ & 343 & 343 & 343 \\
热变形温度 $/{ }^{\circ} \mathrm{C}$ & 163 & 315 & 293 \\
玻璃化转化温度 $/{ }^{\circ} \mathrm{C}$ & 143 & 143 & 143 \\
拉伸强度 $\left(23^{\circ} \mathrm{C}\right) / \mathrm{MPa}$ & 100 & 155 & 134 \\
延伸率 $\left(23^{\circ} \mathrm{C}\right) /(\%)$ & 34 & 2 & 2.2 \\
弯曲强度 $\left(23^{\circ} \mathrm{C}\right) / \mathrm{MPa}$ & 163 & 212 & 186 \\
压缩强度 $\left(23^{\circ} \mathrm{C}\right) / \mathrm{MPa}$ & 118 & 215 & 150 \\
洛氏硬度 $/ \mathrm{D}$ & 84.3 & 89.4 & 86.8 \\
\hline
\end{tabular}

试验选取耐腐蚀的 17-4PH 作为摩擦副材料, 中国牌号为 $0 \mathrm{Cr} 17 \mathrm{Ni} 4 \mathrm{Cu} 4 \mathrm{Nb}$, 是由铜、铌/钶构成的 沉淀、硬化、马氏体不锈钢。经热处理后, 产品的 力学性能更加完善, 可以达到 $1100 \sim 1300 \mathrm{MPa}$ 的 耐压强度, 对稀释酸具有良好的抗腐蚀能力。

\section{2 试验设备及加载}

试验设备：SST-ST 销盘摩擦试验机(德国 Wazau), 试验介质: 人造海水(成分见表 3), 试验时 间为 $2 \mathrm{~h}$, 每种试验重复 2 组。

表 3 人工海水成分

\begin{tabular}{cc||cc}
\hline 成分 & 质量分数 & 成分 & 质量分数 \\
\hline $\mathrm{Na}^{+}$ & 9.92 & $\mathrm{Cl}^{-}$ & 18.01 \\
$\mathrm{Mg}^{2+}$ & 1.16 & $\mathrm{SO}_{4}{ }^{2-}$ & 2.25 \\
$\mathrm{Ca}^{2+}$ & 0.38 & $\mathrm{CO}_{3}{ }^{2-}$ & 0.025 \\
$\mathrm{~K}^{+}$ & 0.36 & $\mathrm{HCO}_{3}{ }^{-}$ & 0.073 \\
\hline
\end{tabular}

PEEK1、PEEK2 和 PEEK3 加工成如图 5b 所 示的销, 使用数控车床加工, 球头表面粗糙度范围

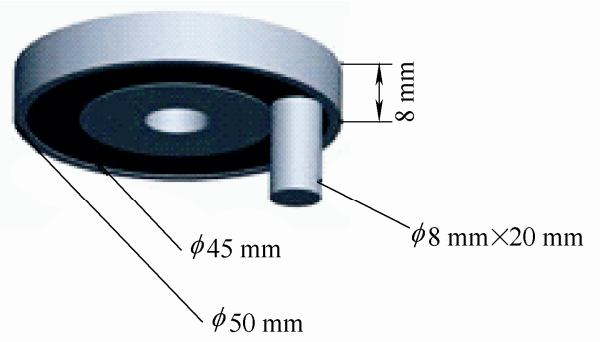

(a) 销盘相对位置
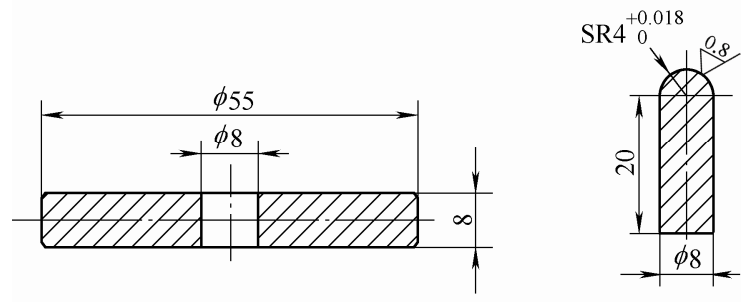

(b) 销盘尺寸

图 5 盘销试验示意图
$0.6 \sim 0.8 \mu \mathrm{m} ; 17-4 \mathrm{PH}$ 加工成图 $5 \mathrm{~b}$ 所示的圆盘，表 面粗䊁度范围 $0.2 \sim 0.4 \mu \mathrm{m}$ 。销盘的相对位置示意 图如图 5a 所示。

试验加载压力为 $20 \mathrm{~N}$, 根据赫兹接触理论计算 结果, 接触应力约为 $160 \mathrm{MPa}$, 符合本文中海水洜 额定载荷; 滑动速度为 $0.5 \mathrm{~m} / \mathrm{s}$, 与微型接触面摩擦 副滚动体额定速度相同。

\section{3 试验结果分析}

图 6 所示为 PEEK1/17-4PH、PEEK2/17-4PH、 $\mathrm{PEEK} 3 / 17-4 \mathrm{PH}$ 摩擦副在人工海水环境中, 滑动速 度为 $0.5 \mathrm{~m} / \mathrm{s}$, 加载压力为 $20 \mathrm{~N}$, 摩擦因数随时间 的变化曲线。PEEK1/17-4PH 摩擦副的摩擦因数在 摩擦初期急剧上升(从 0.08 上升至 0.10 ), 随后随着 时间的增加, 摩擦因数逐渐降低并趋于稳定 $(0.07$ 左右), 整个过程中平均摩擦因数为 0.084 ; PEEK2/17-4PH 摩擦副的摩擦因数在摩擦初期迅速 降低(从 0.19 上升至 0.09 ), 之后随着时间的增加, 摩擦因数有逐渐降低趋势, 并在较大范围内波动 (0.06 0.08 之间), 整个过程中平均摩擦因数为 0.071 , 低于 PEEK1/17-4PH 摩擦副的平均摩擦因 数。PEEK3/17-4PH 摩擦副的摩擦因数整个过程中 比较稳定, 平均值为 0.053 , 比 PEEK $1 / 17-4 \mathrm{PH}$ 摩擦 副的平均摩擦因数低了约 $36.9 \%$ 。

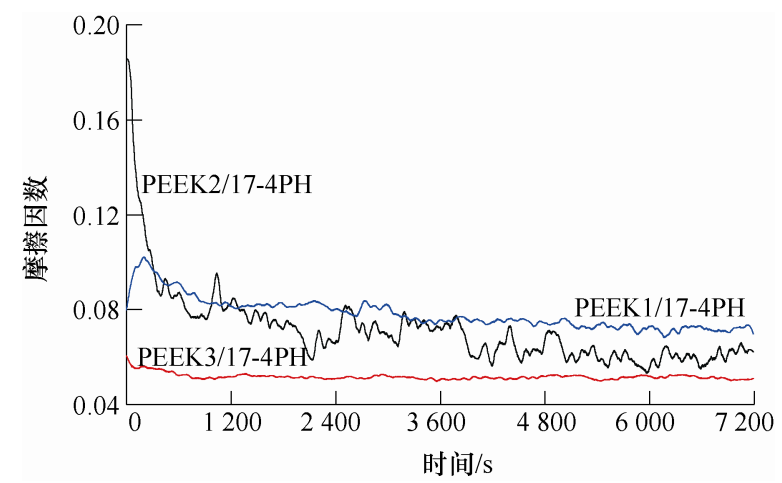

图 6 三种材料在 $20 \mathrm{~N}$ 的压力和 $0.5 \mathrm{~m} / \mathrm{s}$ 速度下摩擦因数

表 4 所示为三种材料平均磨斑直径和磨损体 积。可以看出, 材料 PEEK2 的磨斑直径和磨损体 积最大, 分别为 $2.460 \mathrm{~mm} 、 0.467 \mathrm{~mm}^{3}$; 材料 PEEK1 次之, 材料 PEEK3 的磨斑直径和磨损体积 最小, 分别为 $1.324 \mathrm{~mm} 、 0.039 \mathrm{~mm}^{3}$ 。

\section{表 4 三种材料平均磨斑直径和磨损率}

\begin{tabular}{ccccc}
\hline & $\begin{array}{c}\text { 磨斑直径 } \\
\text { 平均值 } \\
/ \mathrm{mm}\end{array}$ & 误差 & $\begin{array}{c}\text { 磨损体积 } \\
\text { 平均值 } \\
/ \mathrm{mm}^{3}\end{array}$ & 误差 \\
\hline PEEK1 & 2.253 & 0.0035 & 0.326 & 0.0028 \\
PEEK2 & 2.460 & 0.0817 & 0.467 & 0.0643 \\
PEEK3 & 1.324 & 0.1188 & 0.039 & 0.0139 \\
\hline
\end{tabular}


图 7 为三种销试样三维形貌图及盘试样接触表面 轮廓图。图 $7 \mathrm{a}$ 为 PEEK1 与 17-4PH 摩擦后, PEEK1 的表面形貌和 17-4PH 的轮廓图。可以看出, PEEK1 试样表面及 17-4PH 接触区都存在一些明显的犁沟, 并且在 PEEK1 摩擦出口处有材料黏着现象, 磨损机 理主要是磨粒磨损和黏着磨损。 PEEK1 为纯 PEEK材 料, 在摩擦过程中, 硬度较小的 PEEK1 容易受到对 偶件(17-4PH)表面凸起峰刮擦作用而形成犁沟, 造成 磨粒磨损; 同时, PEEK1 被刮擦的材料沿着摩擦方 向不断堆积, 一部分沿着摩擦界面脱落, 另一部分
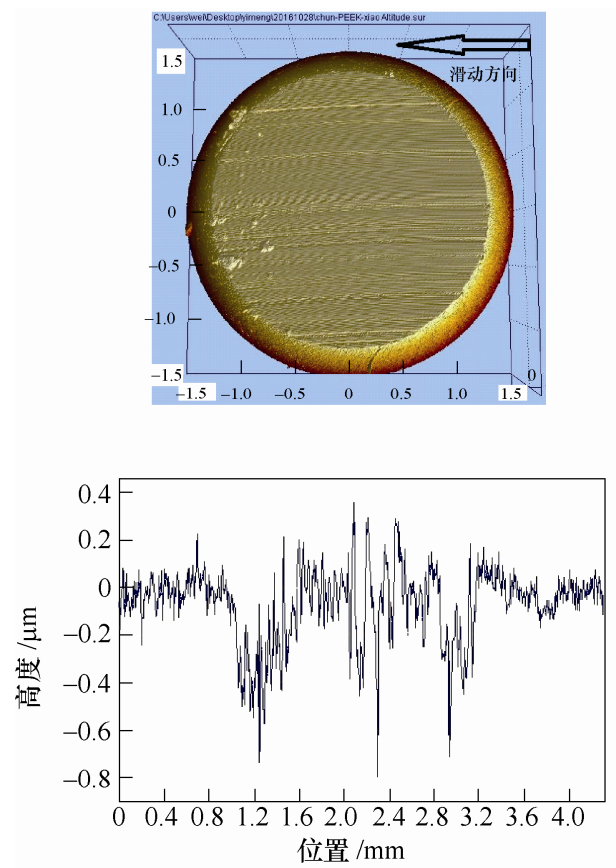

(a) PEEK1与 $17-4 \mathrm{PH}$

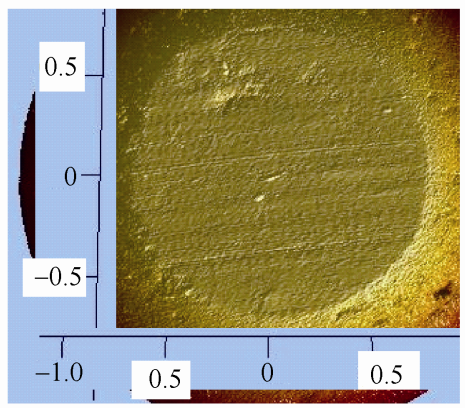

在摩擦力的作用下发生塑性变形, 黍着在试样表面 (如图 7a 所示), 造成黏着磨损。另外，在摩擦的不断 作用下，对偶面出现了许多犁沟，大部分犁沟的深 度约为 $0.5 \mu \mathrm{m}$ 。PEEK 1 与 17-4PH 两表面接触初期, 由于高副接触, 初始接触应力约为 $160 \mathrm{MPa}$, 导致材 料严重的塑性变形和黏着, 所以初期摩擦因数较 大。随着表面的不断磨损, 接触面变大, 接触应力 和摩擦应力不断减少，最终接触应力在 $10 \sim 15 \mathrm{MPa}$ 之间, 塑性变形也因此减少, 摩擦因数随之变小, 并有继续减小的趋势。
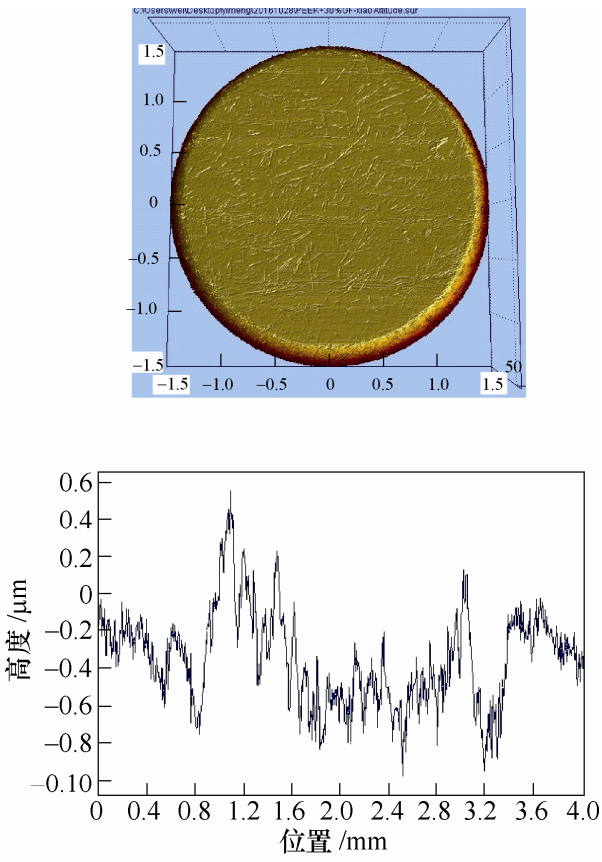

(b) PEEK2 与 $17-4 \mathrm{PH}$

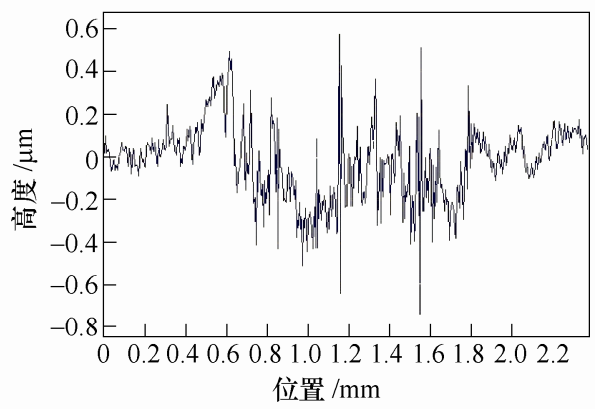

(c) PEEK3与 $17-4 \mathrm{PH}$

图 7 三种销试样三维形貌图及盘试样接触表面轮廓图

图 7b 为 PEEK2 与 17-4PH 摩擦后, PEEK2 的 表面形貌和 17-4PH 的轮廓图。可以看出, PEEK2 摩擦表面有很多不规则坑, 是玻璃纤维在摩擦过程 中受对偶件的黏着作用从基体中剥离, 形成的凹 坑。在摩擦初期, 由于 PEEK2 的拉伸和压缩强度 较大, 塑性变形所需摩擦力大, 所以摩擦力较大, 摩擦因数较高。随着摩擦继续进行, 部分玻璃纤维
从基体中剥离，形成三体磨粒磨损，造成摩擦因数 波动和严重的磨损。PEEK2 和 17-4PH 的主要磨损 机理是粘着磨损和磨粒磨损，磨损在三种材料中最 大。从对偶件轮廓图也可以看出，17-4PH 接触区 波动明显大于其他两种材料, 犁沟深度大部分集中 在 $0.8 \mu \mathrm{m}$ 。

图 7c 为 $\mathrm{PEEK} 3$ 与 $17-4 \mathrm{PH}$ 摩擦后, PEEK3 的 
表面形貌和 17-4PH 的轮廓图。可以看出, PEEK3 磨损表面较为光滑, 沿着运动方向只有少量轻微划 痕。该材料为碳纤、石墨和 PTFE 复合改性 PEEK 材料, 其中石墨和 PTFE 为典型自润滑材料, 用作 改性剂时具有明显的固体润滑作用, 因此, PEEK3 摩擦因数在三种材料中最小, 并且比较稳定。同 时, 碳纤维可以提高基体材料的强度, 加入了 $10 \%$ 碳纤维的 PEEK3 耐磨性能得到显著提高, 磨损低 于其他两种材料。对偶件 17-4PH 接触区犁沟也较 浅, 大部分犁沟深度不到 $0.4 \mu \mathrm{m}$ 。PEEK3/17-4PH 的磨损机理主要是是轻微的磨粒磨损。

\section{3 讨论}

对于微型面接触摩擦副的接触应力和摩擦应力 的计算, Hertz 接触理论没有考虑摩擦力和加速度 等影响, 而在本文模型中, 摩擦因数高达 0.1 , 且存 在多处摩擦副, 所以摩擦力的影响不能忽略, 因此 使用瞬态动力学原理进行计算所得结果更接近实际 工况。

由于海水的冷却作用, 摩擦热量可以不考虑 ${ }^{[15]}$, 因此, 材料本身摩擦磨损性能成为决定微型面接触 摩擦副性能的关键因素。

玻璃纤维和碳纤维都属于基体增强材料。加 入玻璃纤维和碳纤维的 PEEK 材料强度有明显增 强 ${ }^{[16-17]}$ 。但是玻璃纤维在摩擦性能方面较差, 低的 接触应力和相对滑动速度下, 耐磨性几乎没有变 化 ${ }^{[15]}$, 在较高的接触应力下, 磨损明显高于纯聚醚 醚酮材料, 主要因为在摩擦过程中, 玻璃纤维在高 应力的作用下脱落造成三体磨损。而碳纤维具有较 高的承载能力, 加入碳纤维一定程度上可以改善基 体的耐磨性 ${ }^{[17]}$ 。

石墨加入 PEEK 基体中可以降低摩擦因数和磨 损率, 尤其在高温和高负载的工况下, 是目前 PEEK 广泛使用的固体润滑剂。

$\mathrm{CHEN}$ 等 ${ }^{[17]}$ 比较了碳纤维含量从 $5 \%$ 到 $20 \%$ 的 PEEK 的摩擦磨损性能, 当碳纤维含量在 $10 \%$ 时, 磨损率最低。BAHADUR 等 ${ }^{[18]}$ 研究了 PTFE 含量从 $5 \% \sim 20 \%$ 的 PEEK 材料的磨损率, 发现当 PTFE 含 量在 $10 \%$ 时基体的磨损率最低。

所以在聚醚醚酮材料中同时加入 $10 \%$ 石墨、 $10 \%$ 聚四氟乙烯和 $10 \%$ 碳纤维可有效降低摩擦因数 和磨损。

和 PEEK 对偶的金属 17-4PH 表面也有一定的 磨损(见图 7 轮廓), 主要机理是腐蚀。海水中的 $\mathrm{Cl}^{-}$、 $\mathrm{SO}_{4}{ }^{2-}$ 和 $\mathrm{O}_{2}$ 等容易腐蚀金属, 而 $\mathrm{Ca}^{2+}$ 和 $\mathrm{Mg}^{2+}$ 与海水
反应形成沉淀物, 不但阻隔这些离子与金属面接触,

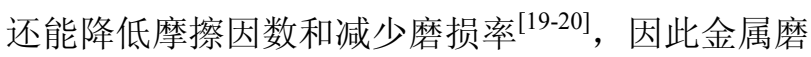
损率极小。本试验只进行了 $2 \mathrm{~h}$, 由于时间较短, 海 水对金属 17-4PH 的腐蚀量很小, 已超出天平的测 量精度，无法测量。

\section{4 结论}

（1）根据柱塞沿缸体运动过程中无刚性冲击和 柔性冲击的准则, 反推出 $\mathrm{B}$ 样条曲线、正弦曲线、 五次多项式曲线, 作为外壳内曲线的轮廓曲线。

(2) 正弦曲线和 B 样条曲线与滚动体组成微型 面接触摩擦副时, 最大接触应力约为 $160 \mathrm{MPa}$, 最 大摩擦应力约为 $16 \mathrm{MPa}$, 接触应力和摩擦应力等指 标均优于多项式曲线。

(3) 纯 PEEK、 $30 \% \mathrm{GF} / \mathrm{PEEK} 、 10 \%$ 石墨 $/ 10 \% \mathrm{CF} /$ $10 \%$ PTFE/PEEK 材料的强度满足要求; 而且经过长 期用, 而腐蚀性等性能已得到验证。

(4) 三组摩擦副中, $10 \%$ 石墨 $/ 10 \% \mathrm{CF} / 10 \% \mathrm{PTFE} /$ PEEK 与 $17-4 \mathrm{PH}$ 摩擦副摩擦因数和磨损量最小, $30 \% \mathrm{GF} / \mathrm{PEEK}$ 与 $17-4 \mathrm{PH}$ 摩擦副磨损量最大, 纯 PEEK 与 $17-4 \mathrm{PH}$ 摩擦副摩擦因数最大; 在 2 小时的 摩擦试验中, 所有对偶件 17-4PH 的磨损量极其微 小, 超出测量精度。

(5) 纯 PEEK 与 17-4PH 摩擦副的磨损机理主 要是黏着磨损和磨粒磨损; $30 \% \mathrm{GF} / \mathrm{PEEK}$ 与 $17-4 \mathrm{PH}$ 摩擦副由于含有玻璃纤维，磨损的主要机理是纤 维脱落后造成的三体磨粒磨损和黏着磨损; $10 \%$ 石墨 $/ 10 \% \mathrm{CF} / 10 \% \mathrm{PTFE} / \mathrm{PEEK}$ 与 $17-4 \mathrm{PH}$ 摩擦副 含有自润滑材料石墨和 PTFE, 以及增强材料碳纤 维, 磨损量仅为 PEEK2 的 $1 / 10$, 机理为轻微的磨 粒磨损。

但是 $10 \%$ 石墨 $/ 10 \% \mathrm{CF} / 10 \% \mathrm{PTFE} / \mathrm{PEEK}$ 含有 $10 \%$ 碳纤维和 $10 \%$ 石墨, 容易和水泵中的金属形成 原电池, 加剧腐蚀磨损, 因此还需要进一步试验考 察其抗腐蚀磨损性能。

\section{参 考 文 献}

[1] 王树杰. 柔性叶片潮流能水轮机水动力学性能研究 [D]. 青岛: 中国海洋大学, 2009 .

WANG Shujie. Study on hydrodynamic performances of a tidal current energy conversion device with flexible blade turbine[D]. Qingdao: Ocean University of China, 2009.

[2] 高殿荣, 王志强, 温茂森, 等. 径向低速大扭矩水液压 马达定子曲线分析 [J]. 燕山大学学报, 2011, 35(6): 493-500.

GAO Dianrong, WANG Zhiqiang, WEN Maosen, et al. 
Analysis of stator curve of low speed high torque water hydraulic motor with radial piston[J]. Journal of Yanshan University, 2011, 35(6) : 493-500.

[3] 黄瑶. 低速大扭矩水压马达受力分析与流场的数值模 拟[D]. 秦皇岛: 燕山大学, 2013.

HUANG Yao. Force analysis and numerical simulation of flow field of low-speed high torque water hydraulic motor[D]. Qinhuangdao: Yanshan University, 2013.

[4] 胡波. 海水径向柱塞泵的研究[D]. 青岛: 中国海洋大 学, 2014.

HU Bo. The reasarch of water hydraulic radial piston pump[D]. Qingdao: Ocean University of China, 2014.

[5] 翟江. 海水淡化高压轴向柱塞泵的关键技术研究 [D]. 杭州: 浙江大学, 2012.

ZHAI Jiang. Investigation into the key problems of axial piston pump for seawater desalination[D]. Hangzhou: Zhejiang University, 2012.

[6] 杨曙东. 基于海水润滑的中高压海水液压泵研究 [D]. 武汉：华中科技大学, 2005 .

YANG Shudong. A dissertation submitted in partial fulfillment of the requirements for the degree of doctor of philosophy in engineering[D]. Wuhan : Huazhong University of Science and Technology, 2005.

[7] YIN F L, NIE S L, ZHANG Z H, et al. Research on the sliding bearing pair of water hydraulic axial piston pump $[\mathrm{J}]$. Journal of Mechanical Engineering Science, 2013, 227(9): 2049-2063.

[8] ZHANG Z H, NIE S L, YUAN S H, et al. Comparative evaluation of tribological characteristics of CF/PEEK and CF/PTFE/Graphite filled PEEK sliding against AISI630 steel for seawater hydraulic piston pumps/motors[J]. Tribology Transactions, 2015, 58: 1096-1104.

[9] 刘恒龙, 柯坚, 王志国, 等. 水压滑靴副的润滑特性 [J]. 机械工程学报, 2006, 42(3): 36-39.

LIU Huanlong, KE Jian, WANG Guozhi, et al. Lubrication characteristics of the water hydraulic sliding shoe pairs[J]. Chinese Journal of Mechanical Engineering, 2006, 42(3): 36-39.

[10] MA L R, LUO J B, ZHANG C H. Effect of solid surface on the formation of thin confined lubricating film of water with micro-content of oil[J]. Applied Surface Science, 2010, 256(22): 6574-6579.
[11] MA L R, LUO J B, ZHANG C H. Film forming characteristics of oil-in-water emulsion with super-low oil concentration[J]. Colloids \& Surfaces A Physicochemical \& Engineering Aspects, 2009, 340(1-3): 70-76.

[12] SUMER M, UNAL H, MIMAROGLU A. Evaluation of tribological behavior of PEEK and glass fiber reinforced PEEK composite under dry sliding and water lubricated conditions[J]. Wear, 2008, 265(7): 1061-1065.

[13] JIA J, CHEN J, ZHOU H, HU L. Comparative study on tribological behaviors of polyetheretherketone composite reinforced with carbon fiber and polytetrafluoroethylene under water-lubricated and dry-sliding against stainless steel[J]. Tribology letters, 2004, 17(2): 231-238.

[14] CHEN B B, WANG J Z, YAN F Y. Comparative investigation on the tribological behaviors of $\mathrm{CF} / \mathrm{PEEK}$ composites under sea water lubrication[J]. Tribology International, 2012, 52(3): 170-177.

[15] UNAL H , MIMAROGL U. Friction and wear characteristics of peek and its composite under water lubrication[J]. Journal of Reinforced Plastics \& Composites, 2006, 25(25): 1659-1667.

[16] CHAIRMAN C, BABU S. Mechanical and abrasive wear behavior of glass and basalt fabric-reinforced epoxy composites[J]. Journal of Applied Polymer Science, 2013, 130(1): 120-130.

[17] PEI X Q, BENNEWITZ R, SCHLARB A. Mechanisms of friction and wear reduction by carbon fiber reinforcement of PEEK[J]. Tribology Letters, 2016，58(3): 1-10.

[18] RODRIGUEZ V, SUKUMARAN J, ALOIS K S, et al. Influence of solid lubricants on tribological properties of polyetheretherketone(PEEK)[J]. Wear, 2016, 103: 45-57.

[19] WANG J Z, YAN F Y, XUE Q J. Tribological behavior of PTFE sliding against steel in sea water[J]. Wear, 2009, 267(9-10): 1634-1641.

[20] REFAIT P, ABDELMOULA M, GENIN J M R, et al. Green rusts in electrochemical and microbially influenced corrosion of steel[J]. Comptes Rendus Geoscience, 2006, 338 (6): 476-487.

作者简介: 周杰(通信作者), 男, 1985 年出生, 博士研究生。主要研究 方向为机械设计及理论。

E-mail: zhou_j09@163.com 